\title{
Resource Allocation with Adaptive Spread Spectrum OFDM Using 2D Spreading for Power Line Communications
}

\author{
Jean-Yves Baudais and Matthieu Crussière \\ Institute for Electronics and Telecommunications of Rennes (IETR), CS 14315, 35043 Rennes, France
}

Received 31 October 2006; Revised 28 February 2007; Accepted 16 May 2007

Recommended by Moisés Vidal Ribeiro

Bit-loading techniques based on orthogonal frequency division mutiplexing (OFDM) are frequently used over wireline channels. In the power line context, channel state information can reasonably be obtained at both transmitter and receiver sides, and adaptive loading can advantageously be carried out. In this paper, we propose to apply loading principles to an spread spectrum OFDM (SS-OFDM) waveform which is a multicarrier system using 2D spreading in the time and frequency domains. The presented algorithm handles the subcarriers, spreading codes, bits and energies assignment in order to maximize the data rate and the range of the communication system. The optimization is realized at a target symbol error rate and under spectral mask constraint as usually imposed. The analytical study shows that the merging principle realized by the spreading code improves the rate and the range of the discrete multitone (DMT) system in single and multiuser contexts. Simulations have been run over measured power line communication (PLC) channel responses and highlight that the proposed system is all the more interesting than the received signal-to-noise ratio (SNR) is low.

Copyright (c) 2007 J.-Y. Baudais and M. Crussière. This is an open access article distributed under the Creative Commons Attribution License, which permits unrestricted use, distribution, and reproduction in any medium, provided the original work is properly cited.

\section{INTRODUCTION}

Different techniques are proposed to provide reliable and high data rate communication access. One of these possible techniques is power line communications (PLC) which exploits the power supply grid for indoor and outdoor communication purpose. Recently, orthogonal frequency division multiplexing (OFDM) has been retained as a good modulation able to ensure high data rates in this frequency selective medium $[1,2]$.

The power line channels essentially offer quasistatic impulse responses, like in other wireline channels, which implies that the channel state information (CSI) can be made available at the transmitter by sending adequate feedback information from the receiver. Under this assumption, the channel knowledge is exploited by bit-loading algorithms to increase the capacity of the transmission systems, as done with the well-know discrete multitone (DMT) system in the digital subscriber line (DSL) applications. This adaptive loading approach results in substantial improvements in terms of system throughput or robustness [3]. In a general approach, each subcarrier can be assigned a given energy and be loaded with a given modulation, such as quadrature am- plitude modulations (QAM). In order to ensure reliable communications, the loading pair constellation energy is driven by the signal-to-noise ratio (SNR) achieved per subcarrier.

However, for long lines or deep fades, the subcarrier SNR can drop under a certain threshold resulting in unload situations. Moreover, finite order constellations like QAM, combined with power spectrum density (PSD) limitations produce a quantification loss that implies a global achievable rate reduction. To circumvent these problems, fractional bit techniques exploiting trellis coded modulations with variable rates can be carried out [4], but lead to an important increase of complexity. Spread spectrum (SS) combined with multicarrier technique has also been proposed using a socalled carrier merging approach $[5,6]$. The merging process consists in connecting a set of subcarriers with spreading sequences. If judiciously done, each resulting set holds an equivalent SNR such that the total supported throughput is greater than the sum of the individual throughputs supported by each subcarrier taken separately. This system, commonly referred to as SS-OFDM, can also be viewed as linear precoded OFDM where the precoded matrix is the spreading matrix [7]. 


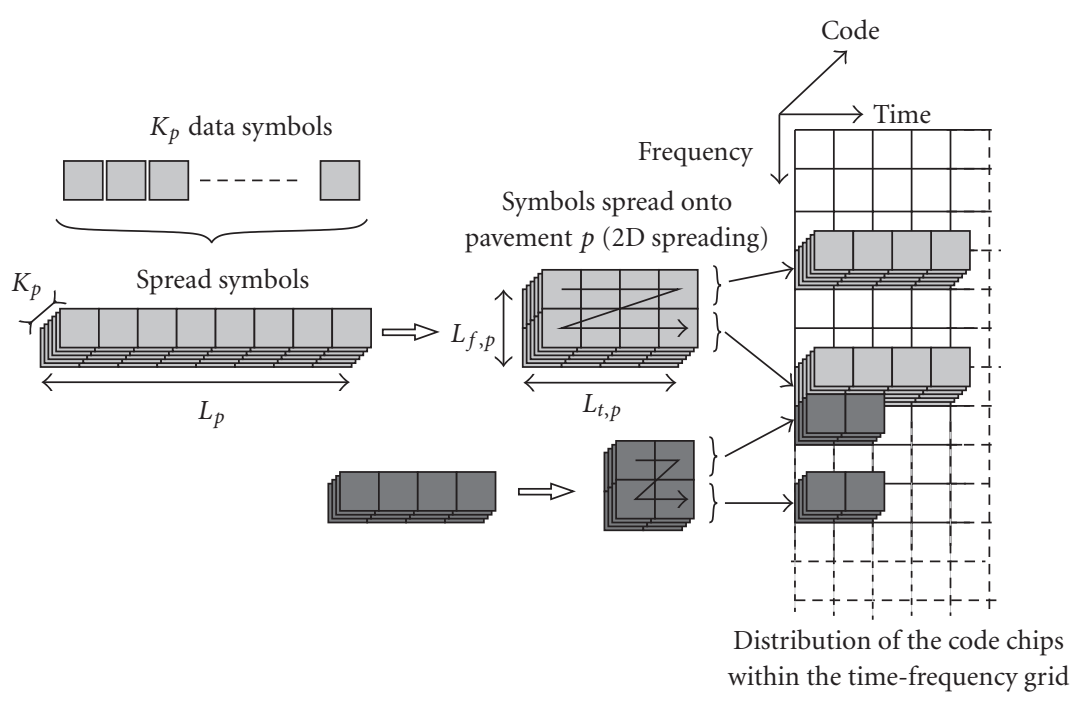

FIgURE 1: Schematic representation of the 2D-spreading technique.

The purpose of this paper is to generalize the above mentioned merging principles exploited for adaptive resource allocation purpose, in the case of 2D time and frequency merging. The related transmission system thus combines OFDM and SS in both domains, time and frequency. Consequently, applying resource allocation to such a system means that the loading algorithm has to take into account not only the subcarriers but also the time and the frequency spreading components of the system to perform bit, energy, and code allocation. Some preliminary works to this study have already been introduced in $[8,9]$ in the case of one dimensional SSOFDM systems. This paper constitutes an overview, a generalization, and an extension to these previous contributions.

This paper is organized as follows. Section 2 presents the SS-OFDM system. Section 3 gives the optimal solution to the throughput maximization problem of the SS-OFDM system within a $2 \mathrm{D}$ time and frequency elementary pavement. Section 4 gives some results with 1D spreading, in time or in frequency, and Section 5 generalizes the spreading in 2D space. Section 6 extends the previous results to the multiuser case. The performance of the proposed scheme is given in Section 7 over power line channels when 2D spreading is applied, in single and multiple user contexts. Finally, Section 8 concludes the paper.

\section{SYSTEM DESCRIPTION}

As previously stated, the studied system results from the combination of multicarrier modulation and spread spectrum. In the general case, the data symbols are spread in time and frequency, and OFDM modulation is applied over the chips of the spreading codes, as presented in [10], thus leading to the 2D SS-OFDM waveform which we are interested in. In our study, the SS component is not used to share access between users, as CDMA does, but instead to multiplex different data symbols belonging to a given user. We then prefer to use the abbreviation SS instead of CDMA. In a multiple user context, developed in Section 6, frequency division multiple access (FDMA) will be used to perform multiple access between users. Figure 1 depicts the construction of the symbol data-flow with respect to the spreading process in time and frequency. As illustrated, the $K_{p}$ data symbols are spread using code sequences of length $L_{p}$. The resulting chips are reshaped into an elementary pavement and are then distributed across the time-frequency grid. The elementary pavement $p$ basically defines the $L_{p}$ chips that are connected by the same codes, and transmitted over a set $\mathcal{L}_{p}$ of $L_{p}$ elements of the time-frequency grid. The distribution is performed over $L_{t, p}$ OFDM symbols and $L_{f, p}$ subcarriers. $L_{t, p}$ and $L_{f, p}$ correspond to the time and frequency spreading factors, respectively, and $L_{t, p} \times L_{f, p}=L_{p}$. The number $P$ of pavements is clearly restricted to be such that $\sum_{p=1}^{P} L_{f, p} \leq N$, where $N$ is the number of available subcarriers of the SS-OFDM system.

The baseband discrete-time equivalent transmitter and receiver model is depicted in Figure 2. The information symbol stream $x_{k, p}(n)$ associated to pavement $p \in[1 ; P]$ is first spread by the code vector $C_{k, p}$ of length $L_{p}$, where $k \in$ $\left[1, K_{p}\right] . K_{p}$ is the number of active codes (see Figure 1), out of a maximum that can be accommodated by the used spreading matrix. With an orthogonal Hadamard matrix $K_{p} \leq L_{p}$, and $L_{p} \in\{1,2,4 i \mid i \in \mathbb{N}\}[11]$, the $K_{p}$ symbols $x_{k, p}(n)$ transmitted over the pavement $p$ are

$$
\begin{aligned}
Y_{p} & =\sum_{k=1}^{K_{p}} C_{k, p} x_{k, p}(n) \\
& =\left[\begin{array}{ccc}
c_{1,1, p} & \cdots & c_{1, K_{p}, p} \\
\vdots & \ddots & \vdots \\
c_{L_{p}, 1, p} & \cdots & c_{L_{p}, K_{p}, p}
\end{array}\right] \times\left[\begin{array}{c}
x_{1, p}(n) \\
\vdots \\
x_{K_{p}, p}(n)
\end{array}\right],
\end{aligned}
$$

where $c_{l, k, p}= \pm 1$ is the code-chip. The chips of signal vector $Y_{p}$ are then distributed within the OFDM timefrequency grid with respect to function $\mathcal{T}$. This function, also called chip-mapping, is handled by the resource 


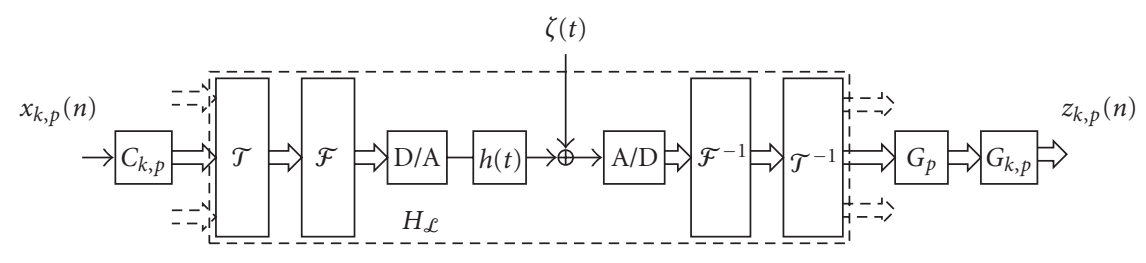

FIgUre 2: Continuous and discrete-time equivalent SS-OFDM model.

allocation algorithm. The resulting data stream is multiplied by the Hermitian Fourier matrix $\mathcal{F}$ that performs the multicarrier modulation. Then digital-to-analog (D/A) conversion yields the continuous-time signal transmitted through the frequency-selective channel $h(t)$.

The received signal is analog-to-digital (A/D) converted and then the multicarrier demodulation $\mathcal{F}^{-1}$ and the dual time-frequency $\mathcal{T}^{-1}$ distribution are applied. The multicarrier component of the SS-OFDM signal is supposed to be adapted to the channel which is assumed to be constant over one SS-OFDM symbol. In that case, the channel can be modeled by one single complex coefficient per subcarrier [12] and represented by a diagonal matrix that takes into account the time and frequency distribution $\mathcal{T}$. Now focusing on a given elementary pavement $p$, that is, on a particular set of elements of the time-frequency grid (see Figure 1), denoted $\mathcal{L}_{p}$, we define the equivalent subchannel matrix $H_{p}$ by

$$
H_{p}=\left[\begin{array}{ccc}
h_{\mathscr{L}_{p}(1)}(n) & & 0 \\
& \ddots & \\
0 & & h_{\mathcal{L}_{p}\left(L_{p}\right)}(n)
\end{array}\right] \text {, }
$$

where $h_{\mathcal{L}_{p}(l)}(n)$ is the frequency channel coefficient of "timesubcarrier" $\mathcal{L}_{p}(l)$. By "time-subcarrier" we mean one subcarrier among the $L_{f, p}$ subcarriers of the elementary pavement $p$, this subcarrier belonging to one of the $L_{t, p}$ OFDM symbols of the SS-OFDM symbol. Before despreading, channel correction based on the zero forcing $(\mathrm{ZF})$ criterion is performed with diagonal matrix $G_{p}$. Hence, diagonal elements of $G_{p}$ are $g_{l, p}=1 / h_{\mathscr{L}_{p}(l)}$. Finally, the received symbol $z_{k, p}(n)$ obtained after despreading using code $C_{k, p}$ writes

$$
z_{k, p}(n)=x_{k, p}(n)+\frac{1}{L_{p}} \sum_{l=1}^{L_{p}} c_{l, k, p} \frac{\zeta_{\mathscr{L}_{p}(l)}(n)}{h_{\mathscr{L}_{p}(l)}(n)},
$$

where $\zeta_{\mathscr{L}_{p}(l)}(n)$ is the sample of complex background noise associated to time-subcarrier $\mathcal{L}_{p}(l)$. This noise is assumed to be Gaussian and white with variance $N_{0}$ for all $l$. Note that if the spreading code is only applied in the time domain, then for all $l \neq l^{\prime} h_{\mathcal{L}(l)}=h_{\mathcal{L}\left(l^{\prime}\right)}$, but $\zeta_{\mathscr{L}(l)} \neq \zeta_{\mathscr{L}\left(l^{\prime}\right)}$. On the other hand, it is important to keep in mind that the SS-OFDM system is reduced to the DMT system when $L_{p}=L_{t, p}=L_{f, p}=1$.

To make the notation more compact and without loss of generality, the time variable $n$ is omitted in the following.

\section{THROUGHPUT MAXIMIZATION}

The proposed SS-OFDM system offers many degrees of freedom which are the code length, the number of codes, the time and frequency spreading factors, the number of bits per code, and the energy per code. In a general approach, these degrees of freedom define variable parameters that can be adjusted to manage resource allocation and maximize the throughput of the system. Let us first focus on the optimal resource allocation within a given elementary pavement $p$ of the SS-OFDM system. The optimal allocation of bits, energies, spreading factors, and codes has to be found considering a particular set of subcarriers $\mathcal{L}_{p}$ such that $\left|\mathcal{L}_{p}\right|=L_{p}$ and under PSD constraint. In this section, one single elementary pavement is considered and the subscript $p$ is omitted.

\subsection{Rate upper-bound}

A rate upper-bound of the system can be derived by evaluating the system capacity which takes into account the channel, the used waveform, and the receiver structure. The system capacity is derived from the mutual information of the SS-OFDM system. It has been proved in [7] that optimal waveform capacity is obtained with Hadamard matrices as spreading matrices. Due to orthogonality, each received symbol $z_{k}$ is estimated independently without intersymbol interference, as evident from (3). Thus, the total system capacity is the sum of the system capacities associated with each code $k$. This total system capacity, expressed in bit per SS-OFDM symbol, with ZF detection is then

$$
\mathcal{C}=\sum_{k=1}^{K} \log _{2}\left(1+\frac{L^{2}}{\sum_{l \in \mathcal{L}}\left(1 /\left|h_{l}\right|^{2}\right)} \frac{e_{k}}{N_{0}}\right),
$$

where $e_{k}$ is the energy associated to the code $k$. The energy $e_{k}$ has to respect the PSD constraint expressed as

$$
\forall k \in[1 ; K], \quad \sum_{k=1}^{K} e_{k} \leq E,
$$

where $E$ is given by the maximal PSD.

Applying classical Lagrange optimization to concave function $C$ in (4) under PSD constraint (5) leads to the following theorem which gives the maximal system capacity.

Theorem 1. With ZF detection, the maximal SS-OFDM system capacity using code length $L$ and a set $\mathcal{L}$ of subcarriers is

$$
\mathcal{C}=L \log _{2}\left(1+\frac{L}{\sum_{l \in \mathcal{L}}\left(1 /\left|h_{l}\right|^{2}\right)} \frac{E}{N_{0}}\right) .
$$


The fairly simple solution stated in Theorem 1 consists in achieving a uniform distribution of energies between the $L$ available codes, that is, for all $k \in[1 ; L], e_{k}=E / L$. Note that this result implicitly says that all of the available codes must be exploited to ensure maximal capacity, that is, $K=L$.

In order to work on a throughput bound rather than on a capacity bound, a convenient quantity called the signalto-noise ratio gap $\Gamma$, sometimes called the normalized $S N R$, is introduced. This gap is a measure of the loss introduced by the QAM with respect to theoretical optimum capacity. With channel coding, the SNR gap is modified to include the coding gain and can also include an additional noise margin which takes into account the impairments of the system [3]. The maximal SS-OFDM throughput $\mathcal{R} \in \mathbb{R}$ for one elementary pavement is then

$$
\mathcal{R}=L \log _{2}\left(1+\frac{1}{\Gamma} \frac{L}{\sum_{l \in \mathscr{L}}\left(1 /\left|h_{l}\right|^{2}\right)} \frac{E}{N_{0}}\right) .
$$

This throughput is the rate upper-bound of the SSOFDM system and will be referred to as such in the remainder of the paper.

\subsection{Discrete modulations}

The above obtained optimal allocation leads to noninteger modulation orders except in the particular case of $\lfloor\mathcal{R} / L\rfloor=$ $\mathcal{R} / L$. Hence, Theorem 1 cannot be applied in practice, and workable rates have to be considered. Denoting $R_{k}$ the rate associated with code $k$, the total throughput of the system can be decomposed as

$$
R=\sum_{k=1}^{K} R_{k}=\sum_{k=1}^{K} \log _{2}\left(1+\frac{1}{\Gamma} \frac{L^{2}}{\sum_{l \in \mathcal{L}}\left(1 /\left|h_{l}\right|^{2}\right)} \frac{e_{k}}{N_{0}}\right)
$$

and in the case of integer order modulations, the following theorem gives the optimal allocation.

Theorem 2. With ZF detection and integer order QAM, the maximal reliable throughput of SS-OFDM using code length $L$, a set $\mathcal{L}$ of subcarriers, and with a rate upper-bound $\mathcal{R}$ is obtained with $\lfloor\mathcal{R} / L\rfloor+1$ bits assigned to $\left\lfloor L\left(2^{\mathcal{R} / L-\lfloor\mathcal{R} / L\rfloor}-1\right)\right\rfloor$ codes and $\lfloor\mathcal{R} / L\rfloor$ bits assigned to $L-\left\lfloor L\left(2^{\mathcal{R} / L-\lfloor\mathcal{R} / L\rfloor}-1\right)\right\rfloor$ codes.

The details of the proof are given in [9], and are based on simple analytical tools. This proof basically shows (i) that the proposed bit distribution among the codes is the one that costs minimum energy, and (ii) that the given throughput is the maximal throughput that is achieved respecting the energy constraint (5). From (5) and (8) this energy cost expresses

$$
\sum_{k=1}^{L} e_{k}=\frac{\Gamma N_{0}}{L^{2}} \sum_{l \in \mathcal{L}} \frac{1}{\left|h_{l}\right|^{2}} \times \sum_{k=1}^{L}\left(2^{R_{k}}-1\right) \leq E .
$$

Theorem 2 simply says that maximal throughput is reached when bits and energies are distributed as uniformly as possible across the codes. The optimal reliable throughput then writes

$$
R=\sum_{k=1}^{L} R_{k}=L\lfloor\mathcal{R} / L\rfloor+\left\lfloor L\left(2^{\mathcal{R} / L-\lfloor\mathcal{R} / L\rfloor}-1\right)\right\rfloor
$$

and the throughput $R_{k}$ per code are such that $R_{k} \in$ $\{\lfloor\mathcal{R} / L\rfloor,\lfloor\mathcal{R} / L\rfloor+1\}$. This theorem also gives the number $K$ of codes to use which is $L$ if $\lfloor\mathcal{R} / L\rfloor \neq 0$, and $\left\lfloor L\left(2^{\mathcal{R} / L}-1\right)\right\rfloor$ otherwise.

\section{1D SPREADING CASE}

Previous results are given for one elementary pavement $p$. In this section we apply the previous results with time or frequency spreading, and for multiple pavements $p \in[1 ; P]$. In a general approach, each elementary pavement can exploit its own code length $L_{p}$ which also becomes an adaptive parameter. But finding the optimal code lengths amounts to resolving a complex combinatorial optimization problem that cannot be reduced to an equivalent convex problem. Then, no analytical solution exists and optimal solution can only be obtained following exhaustive search. In order to avoid prohibitive computations, we assume that all the codes have the same length $L$ independently of $p$, such that $L=L_{t} \times L_{f}$. This suboptimal but simple solution gives very satisfying results compared to the adaptive code length solution [7]. Furthermore, using unique code length allows to derive useful analytical results. In the sequel, let then $L_{p}=L, L_{t, p}=L_{t}$, and $L_{f, p}=L_{f}$ for all $p$ in $[1 ; P]$.

\subsection{Time spreading}

In the case of $1 \mathrm{D}$ time spreading $L_{t}=L$, the system would then be an multicarrier direct sequence code division multiple access (MC-DS-CDMA) system if the spreading component were used to realize multiple access between users [13]. Subcarrier coefficients $h_{l, p}$ of one elementary pavement $p$ are equal, since the channel is unchanged over one SS-OFDM symbol. The throughput in $\mathbb{R}$ then writes

$$
\mathcal{R}_{p}=L \log _{2}\left(1+\frac{1}{\Gamma}\left|h_{l, p}\right|^{2} \frac{E}{N_{0}}\right)
$$

and the throughput $\mathcal{R}$ of an SS-OFDM system using $N$ subcarriers, or $P=N$ elementary pavements, is simply the sum over $N$ of the throughputs $\mathcal{R}_{p}$ of each elementary pavement $p$. Throughput $R_{p}$ is then simply expressed replacing $\mathcal{R}$ by $\mathcal{R}_{p}$ in (10).

As evident from (11) and (10), $\mathcal{R}_{p}(L)$ and $R_{p}(L)$ are increasing functions. Expressed in bit per OFDM symbol, the reliable rate $R_{p} / L$ can reach the rate upper-bound per OFDM symbol $\mathcal{R}_{p} / L$ if and only if $\mathcal{R}_{p} / L$ is integer, which is stated in the following proposition.

Proposition 1. If $\left\lfloor\mathcal{R}_{p} / L\right\rfloor \neq \mathcal{R}_{p} / L$, then for all $L R_{p}<\mathcal{R}_{p}$. 


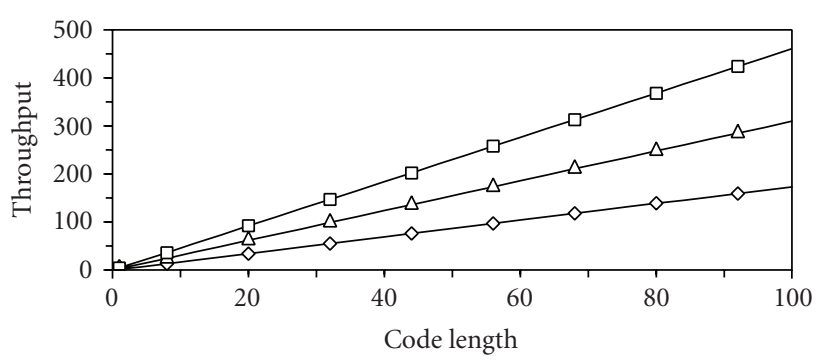

$\square 20(\mathrm{~dB})$

$\triangle 15(\mathrm{~dB})$

$\diamond 10(\mathrm{~dB})$

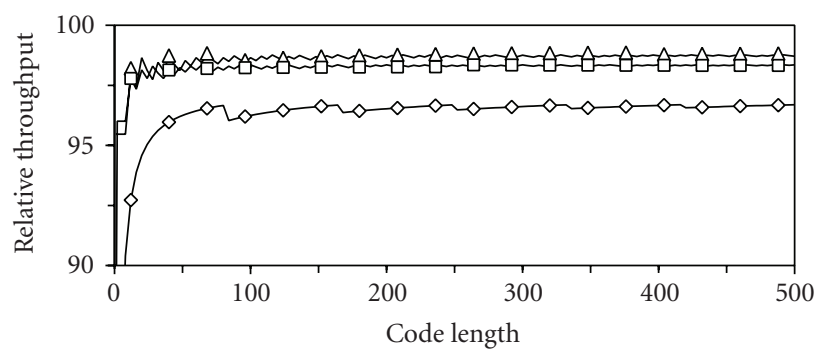

$\square 20(\mathrm{~dB})$

$\triangle 15(\mathrm{~dB})$

$\diamond 10(\mathrm{~dB})$

FIgURE 3: Throughput $R_{p}$ in bit per SS-OFDM symbol and relative throughput $R_{p} / \mathcal{R}_{p}$ in percent versus code length $L_{t}=L$, for 1 subcarrier, 3 received SNR $\{10,15,20\} \mathrm{dB}$ and $\Gamma=6 \mathrm{~dB}$.

Proof. It is simply proved using that if $x \in \mathbb{R}$ and $x \neq\lfloor x\rfloor$, then $2^{x-\lfloor x\rfloor}-1<x-\lfloor x\rfloor$. Thus

$$
\begin{aligned}
R_{p} & =L\left\lfloor\frac{\mathcal{R}_{p}}{L}\right\rfloor+\left\lfloor L\left(2^{\mathcal{R}_{p} / L-\left\lfloor\mathcal{R}_{p} / L\right\rfloor}-1\right)\right\rfloor \\
& \leq L\left\lfloor\frac{\mathcal{R}_{p}}{L}\right\rfloor+L\left(2^{\mathcal{R}_{p} / L-\left\lfloor\mathcal{R}_{p} / L\right\rfloor}-1\right) \\
& <\mathcal{R}_{p} .
\end{aligned}
$$

This inequality is true for all $L \in \mathbb{N}^{*}$.

To illustrate Proposition 1, Figure 3 shows that the throughput $R_{p}$ is an increasing function of $L$, whereas the relative throughput $R_{p} / \mathcal{R}_{p}$ converges to a value inferior to $100 \%$. In this figure, the received SNR equals $\left|h_{l, p}\right|^{2}\left(E / N_{0}\right)$. This relative throughput is overall increasing with $L$, but can locally decrease due to the integer part operator.

We have introduced a rate upper-bound in Section 3.1 which gives a bound in $\mathbb{R}$ of a reliable rate in $\mathbb{N}$. This bound is actually not a reachable rate in general as it can be viewed in Figure 3. Proposition 1 then induces a new upper-bound well suited for reliable throughputs defined in $\mathbb{N}$. Let $\mathfrak{R}_{p}$ be this new reliable upper-bound:

$$
\mathfrak{R}_{p}=\lim _{L \rightarrow \infty} \frac{R_{p}}{L}=\left\lfloor\frac{\mathcal{R}_{p}}{L}\right\rfloor+2^{\mathcal{R}_{p} / L-\left\lfloor\mathcal{R}_{p} / L\right\rfloor}-1 .
$$

This upper-bound is then expressed in bit per OFDM symbol, and combining (11) with (13) it is clear that $\mathfrak{R}_{p}$ is independent of $L$.

In terms of system performance, Figure 3 shows that the time spreading exploits energy merging to improve the throughput. Indeed, the SS-OFDM throughput with $L_{t}=$ $L>1$ cannot be lower than that obtained with $L_{t}=1$ which corresponds to the DMT system. The difference between the throughput $R_{p}$ and the rate upper-bound $\mathcal{R}_{p}$ is reduced which means that the energy merging translates into a compensation of the energy loss brought by the integer order modulations.

\subsection{Frequency spreading}

With 1D time spreading, the energy merging is realized between the same time-subcarriers of several successive OFDM symbols. With 1D frequency spreading, the merging is instead realized between different subcarriers belonging to the same OFDM symbol. One SS-OFDM symbol is then reduced to one OFDM symbol, and the corresponding system would be an MC-CDMA (multicarrier coded division multiple access) system if the spreading component were used to realize multiple access between users [14]. In a general approach, the gains of the subcarriers over a subset $\mathcal{L}_{p}$ are not equal, that is, for all $\left\{l, l^{\prime}\right\} \in \mathcal{L}_{p}^{2}$ and $l \neq l^{\prime},\left|h_{l, p}\right|^{2} \neq\left|h_{l^{\prime}, p}\right|^{2}$. Then, the rate upper-bound $\mathcal{R}_{p}$ of pavement $p$ is directly given by (7) without any simplifications, and the achievable throughput $R_{p}$ is simply expressed replacing $\mathcal{R}$ by $\mathcal{R}_{p}$ in (10).

This SS-OFDM system can benefit from carrier merging to improved the system throughput even if the subcarriers have different gains. For example, let $P=2$, and $\left|\hat{h}_{i}\right|^{2}=\left|h_{i}\right|^{2}\left(E / \Gamma N_{0}\right)$, with $\left|\hat{h}_{1}\right|^{2}=3.4$ and $\left|\hat{h}_{2}\right|^{2}=2.9$. The DMT throughput corresponding to this two-subcarrier system equals 3 whereas the SS-OFDM throughput equals 4:

$$
\begin{gathered}
R_{\mathrm{DMT}}=\left\lfloor\log _{2}(1+3.4)\right\rfloor+\left\lfloor\log _{2}(1+2.9)\right\rfloor=3, \\
R_{\mathrm{SS}-\mathrm{OFDM}}=2 \times\left\lfloor\log _{2}(1+3.13)\right\rfloor+0=4 .
\end{gathered}
$$

The throughput gain is expected to be all the more important than the amount of merged energies is high, that is, the frequency spreading factor increases. However, merging subcarriers with variable gains also leads to distortion within pavements. The ZF detector suppresses this distortion restoring the code orthogonality to the detriment of a noise level enhancement.

As it is shown in Figure 4 over uncorrelated Rayleigh fading channel of 100 subcarriers, the throughput overall increases with the code length but when the system exploits subcarriers with small received SNR, the carrier merging cannot compensate for the distortion. This is the case, for example, when $h_{30}$ or $h_{40}$ are exploited with code lengths, respectively, equal to 30 and 40 . For small code lengths, the throughput $R_{p}$ is very far from upper-bound $\mathcal{R}_{p}$, whereas the difference $R_{p} / \mathcal{R}_{p}$ reaches a minimum for code lengths around 10-40 as evident from the relative throughput curve in Figure 4. Now focusing on the code throughput $R_{p} / K$, it is shown that the number of bit per code overall decreases with 

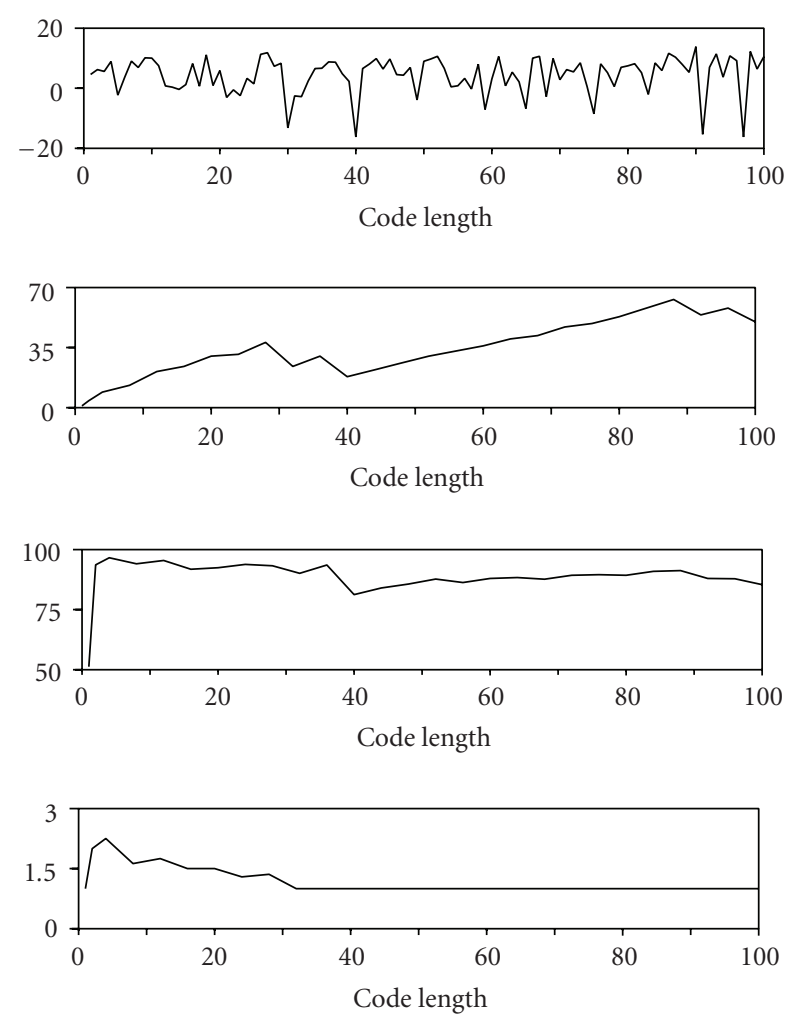

Figure 4: Received SNR in dB per subcarrier (a), throughput $R_{p}$ in bit per SS-OFDM symbol (b), relative throughput $R_{p} / \mathcal{R}_{p}$ in $\%$ (c), and code throughput $R_{p} / K$ (d) versus code length $L_{f}=L$ over 100 subcarriers, $\Gamma=6 \mathrm{~dB}$.

code length. This means that the gain brought by the energy merging cannot compensate for the distortion.

A solution to mitigate the distortion is to use multiple elementary pavements thus reducing the spreading factor while exploiting the highest possible number of subcarriers. The corresponding system is commonly called spreadspectrum multi-carrier multiple access (SS-MC-MA) system [15]. Such a system needs to distribute subcarriers between the elementary pavements. The following proposition gives the optimal distribution that maximizes the throughput $\mathcal{R}$.

Proposition 2. The optimal subcarrier subsets $\mathcal{L}_{p}, p \in[1 ; P]$, that maximize the throughput $\mathcal{R}=\sum_{p=1}^{P} \mathcal{R}_{p}$ are such that for all $p \neq p^{\prime}$, for all $l \in \mathcal{L}_{p}$, for all $l^{\prime} \in \mathcal{L}_{p^{\prime}}$, then $\left|h_{l}\right| \geq\left|h_{l^{\prime}}\right|$.

Proof. We have basically shown that any subcarriers swapping between two subsets $\mathscr{L}_{p}$ and $\mathscr{L}_{p^{\prime}}$ given by the proposition leads to a rate loss. This is done using simple derivation study of a sum of logarithm functions. The result obtained for two subsets is easily generalized for $P$ subsets.

A practical solution to make use of Proposition 2 is to sort the subcarriers in descending order of power gain. The same result is obtained by symmetric with ascending order and also minimizes the distortion within each subset and maximizes the total throughput $\mathcal{R}$. However, Proposition 2
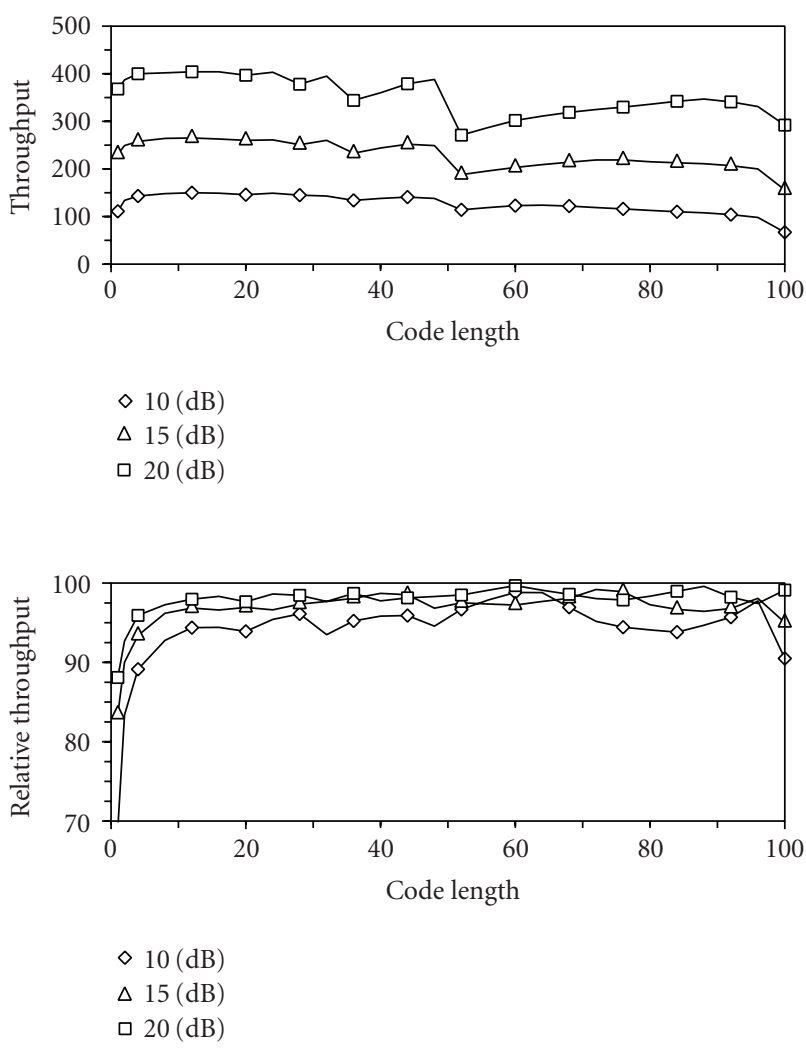

Figure 5: Throughput $R$ in bit per SS-OFDM symbol and relative throughput $R / \mathcal{R}$ in percent versus code length $L_{f}=L$ over 100 subcarriers, for 3 average SNR $\{10,15,20\} \mathrm{dB}$, and $\Gamma=6 \mathrm{~dB}$.

which gives the unique optimal subcarrier distribution between subsets for $\mathcal{R} \in \mathbb{R}$, only yields a suboptimal solution to the $R \in \mathbb{N}$ maximization problem. The optimal subset choice for $R \in \mathbb{R}$ would consist in finding the subcarriers that fully exploit the PSD in each subset. This optimal solution could be obtained following a subcarrier swapping approach after initial subcarrier distribution given by Proposition 2 . The resulting algorithm would require a prohibitive intensive computation and the resulting rate gain would however not be sufficiently high to compensate for this complexity increase [9]. Therefore, we directly exploit Proposition 2 to distribute subcarriers and then simply apply Theorem 2 in each resulting pavement to maximize $R$ with low complexity cost.

Figure 5 gives throughput $R=\sum_{p} R_{p}$ and relative throughput $R / \mathcal{R}$ of the SS-OFDM system over uncorrelated Rayleigh fading channel of 100 subcarriers. The average SNR is given by

$$
\mathrm{E}\left[\left|h_{i}\right|^{2} \frac{E}{N_{0}}\right]=\frac{1}{100} \sum_{i=1}^{100}\left|h_{i}\right|^{2} \frac{E}{N_{0}} .
$$

All the 100 subcarriers are used if and only if the code length $L_{f}$ is a divider of 100. In spite of this disadvantage, the throughput increases with $L_{f}$ up to $L_{f} \approx 20$. For $L_{f} \geq 52$, the system is composed of only one elementary pavement and can then exploit only $L_{f}$ subcarriers. In that case, the 


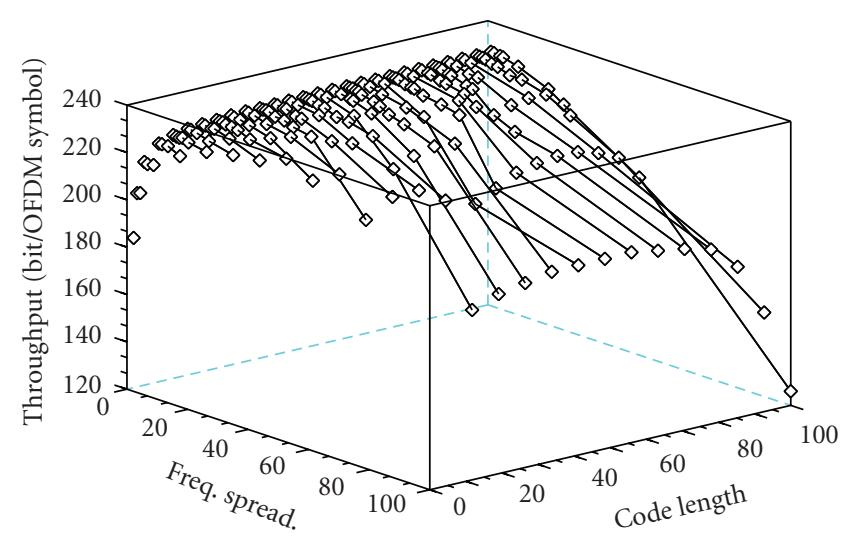

Figure 6: Throughput $R / L_{t}$ versus code length $L$ and frequency spreading factor $L_{f}$ over 100 subcarriers, with an average SNR of $20 \mathrm{~dB}$ and $\Gamma=6 \mathrm{~dB}$.

obtained throughputs are lower than those obtained with lower code lengths. As in Figure 3, for small code lengths, the throughput $R_{p}$ is very far from upper-bound $\mathcal{R}_{p}$, whereas this difference reduces for higher code lengths. Figure 5 also shows that $L_{f}=1$ is not the optimal code length configuration for throughput in $\mathbb{N}$. However, the optimal configuration cannot be derived analytically.

Note that a more powerful equalizer such as minimum mean square error (MMSE) equalizer rather than ZF equalizer could have been chosen to mitigate noise effect. However, Proposition 2 leads to subcarrier distribution that minimizes channel distortion - and noise effect-within each subset. Then, ZF detection leads to throughputs very close to those obtained with MMSE equalizer. Furthermore, mathematical expressions obtained with MMSE have a form such that the studied optimization problem is not convex, whereas derivations with ZF are fairly simple and lead to a closed form solution to the optimization problem.

\section{2D SPREADING OPTIMIZATION}

Merging the results in Section 4 obtained with 1D time or frequency spreading, the throughput per elementary pavement writes with $2 \mathrm{D}$ time and frequency spreading

$$
\mathcal{R}_{p}=L \log _{2}\left(1+\frac{1}{\Gamma} \frac{L_{f}}{\sum_{l=1}^{L_{f}}\left(1 /\left|h_{l, p}\right|^{2}\right)} \frac{E}{N_{0}}\right),
$$

and applying (10) the total reliable throughput yields

$$
R=\sum_{p=1}^{P} L\left\lfloor\frac{\mathcal{R}_{p}}{L}\right\rfloor+\sum_{p=1}^{P}\left\lfloor L\left(2^{\mathcal{R}_{p} / L-\left\lfloor\mathcal{R}_{p} / L\right\rfloor}-1\right)\right\rfloor .
$$

Figure 6 gives reliable throughput $R / L_{t}$ per OFDM symbol of the SS-OFDM system over uncorrelated Rayleigh fading channel of 100 subcarriers. It firstly appears that the throughput increases with $L_{t}$, that is, with $L$ for a fixed value of $L_{f}$, as already mentioned in Figure 3, and quickly reaches its maximal value. On the other hand, for a fixed spreading factor $L$, the throughput degrades for high values of $L_{f}$ due to the increase of the frequency distortion. If the maximal time spreading factor $L_{t}$ is only limited by $L$, the highest throughputs are obtained for small values of $L_{f}$ and for high values of $L_{t}$. Such configurations minimize the distortion within 2D pavements. The DMT throughput given by $L=L_{t}=L_{f}=1$ is around 130 bits per OFDM symbol, and is then easily outperformed by the SS-OFDM system.

It turns out that the optimal configuration, that is, code length, time, and frequency spreading factors, cannot be reached analytically for throughput in $\mathbb{N}$, whereas the following proposition gives the optimal configuration in $\mathbb{R}$.

Proposition 3. The throughput $\mathcal{R}=\sum_{p=1}^{P} \mathcal{R}_{p}$ is maximal for $L_{f}=1$.

Proof. The case of two subcarriers and $L_{f} \in\{1 ; 2\}$ is analyzed, the generalization being obvious. Let $x, y$ be the two normalized SNR per subcarrier. The throughputs write

$$
\begin{aligned}
& \mathcal{R}_{\left(L_{f}=1\right)}=L_{t} \log _{2}(1+x)+L_{t} \log _{2}(1+y), \\
& \mathcal{R}_{\left(L_{f}=2\right)}=L_{t} \times 2 \log _{2}\left(1+\frac{2}{1 / x+1 / y}\right) .
\end{aligned}
$$

The difference between these two functions shows that $\mathcal{R}_{\left(L_{f}=2\right)} \leq \mathcal{R}_{\left(L_{f}=1\right)}$ for all $L_{t}$.

The reliable rate upper-bound $\Re_{p}$ introduced in Section 4.1 remains valid using $\mathcal{R}_{p}$ defined in (16) and (13). $\Re_{p}$ then depends on the frequency spreading factor $L_{f}$ and, contrary to $\mathcal{R} \in \mathbb{R}$ given by Proposition 3 , we cannot obtain any analytical total reliable upper-bound $\Re \in \mathbb{N}$ independent of $L_{f}$.

\section{MULTIUSER EXTENSION}

In the single user context, the bit-loading algorithm applies Theorem 2 to perform bit, code, and energy allocation across the spectrum.

In the multiuser context different resource sharing strategies can be used (see, e.g., $[16,17]$ and related references). We choose here to maximize the smallest throughput over all users, which is equivalent to maximize the total throughput of the system while ensuring equal rates between users. This strategy then sacrifices the overall performance of the system, measured as the sum rate of all active users. Maximizing this sum rate can be done at low computational complexity but favors only users with good channels and does not ensure bandwidth for all users. Maximizing the minimum throughput ensures minimum quality of services for all the active users, and then guarantees fairness between them.

To realize multiple access, we use a modified version of the subcarrier allocation algorithm proposed in [18]. Let $N_{u}$ be the number of users, $R^{(u)}$ the throughput of user $u$, and $\mathcal{B}_{u}$ the subset of subcarriers used by user $u$ that gathers several elementary pavements. Because of spectrum sharing between users following FDMA, we have for all $u \neq u^{\prime}, \mathscr{B}_{u} \cap \mathscr{B}_{u^{\prime}}=\varnothing$. For given $L, L_{f}$, and $L_{t}$, the proposed allocation Algorithm 1 is realized in three steps [9]. 
(1) Initialization

(a) Compute $\forall u \quad \alpha_{u}=R^{(u)}$, with $\mathscr{B}_{u}=[1 ; N]$

(b) Set $\forall u R^{(u)}=0, \mathcal{B}_{u}=\varnothing$

(2) While $\exists u, \mathscr{B}_{u}=\varnothing$
(a) Find $u=\operatorname{argmin}_{u}\left\{\alpha_{u} \mid \mathscr{B}_{u}=\varnothing\right\}$
(b) For the found $u$, find the best unused $L_{f}$ subcarriers
(c) Update $\mathscr{B}_{u}, R^{(u)}, \alpha_{u}$

(3) While there exists unused subcarrier
(a) Find $u=\operatorname{argmin}_{u}\left\{R^{(u)} \mid \exists p, R_{p}^{(u)}>0\right\}$
(b) For the found $u$, find the best unused $L_{f}$ subcarriers
(c) Update $\mathscr{B}_{u}, R^{(u)}$.

\section{Algorithm 1}

The modifications of the algorithm proposed in [18] are as follows (i) subcarriers are allocated to users by sets of $L_{f}$ subcarriers instead of being allocated one by one; (ii) the $N_{u}$ first sets of subcarriers are assigned in step 2 with respect to a priority order among the users based on the achievable throughput of each user computed over all the available subcarriers; and (iii) the user which cannot improve its rate is no more taken into account by the allocation procedure in step 3. The user with the smallest $R^{(u)}$ is being allocated at first, and then are the others. Of course step 3 is stopped when no more user can improve its throughput. Without condition in 3(a), the user with the worst channel would impose its throughput on all the other users, which would reduce the total throughput. Note that the structure of the algorithm is independent of the code length $L$ and can be applied for $L=1$ as well as for $L>1$.

\section{SIMULATION RESULTS OVER POWER LINE CHANNELS}

In practical systems, there is $P=\left\lfloor N / L_{f}\right\rfloor$ elementary pavements over $N$ subcarriers then

$$
\mathcal{R}=\sum_{p=1}^{P} \mathcal{R}_{p}, \quad \mathfrak{R}=\sum_{p=1}^{P} \mathfrak{R}_{p}, \quad R=\sum_{p=1}^{P} R_{p},
$$

and in order to compare the performance of the systems, the throughputs are given in bit per OFDM symbol, that is, these throughputs are $\mathcal{R} / L_{t}, \mathfrak{R}$, and $R / L_{t}$.

In this section, we present simulation results for the proposed adaptive SS-OFDM scheme and we compare the performance of the new scheme with the performance of DMT, that is, when $L=L_{f}=L_{t}=1$. The generated SSOFDM signal is composed of 2048 subcarriers transmitted in the band $[0 ; 20] \mathrm{MHz}$, and 1880 subcarriers are used to transmit information data. Then $N=1880$ and the resulting used bandwidth is $[1.6 ; 20] \mathrm{MHz}$. The subcarrier spacing equals $9.765 \mathrm{kHz}$ and a long enough cyclic prefix is used to overcome intersymbol interference. We assume that the

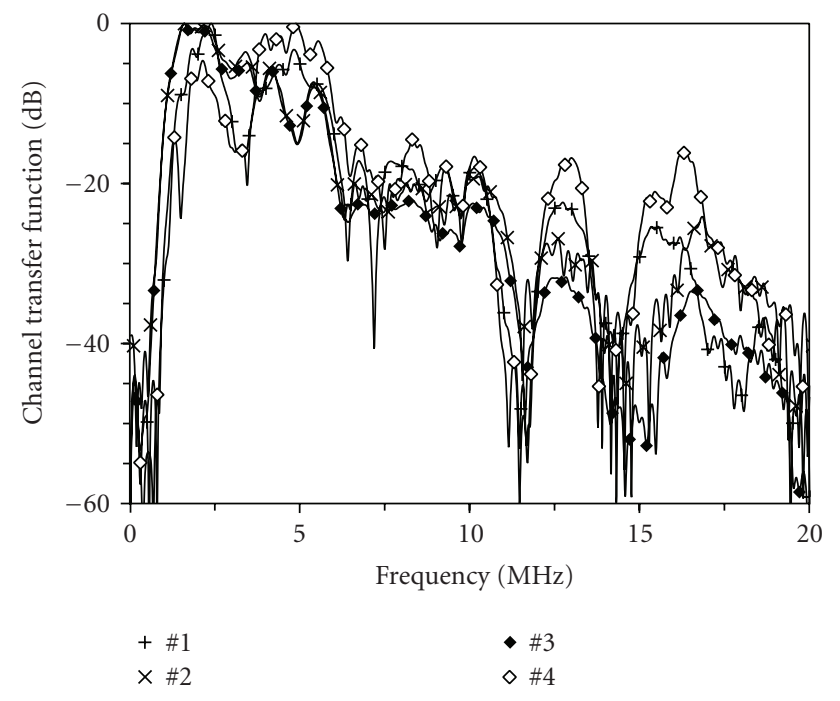

FIgURE 7: Measured PLC channel transfer functions.

synchronization and channel estimation tasks have successfully been treated. The used PLC responses, displayed in Figure 7, have been measured in an outdoor residential network by the French power company Electricité de France (EDF). We assume a background noise level of $-110 \mathrm{dBm} / \mathrm{Hz}$ and the signal is transmitted with respect to a maximal PSD of $-40 \mathrm{dBm} / \mathrm{Hz}$. We consider that $2^{q}$-ary QAM are employed with $q \in[2 ; 15]$ as in DSL specifications. Results are given for a target symbol error rate (SER) of $10^{-3}$ corresponding to an SNR gap $\Gamma=6 \mathrm{~dB}$ without channel coding. Some results are given versus channel attenuation which is related to maximal received SNR per subcarrier in the following way:

$$
\max \mathrm{SNR}_{(\mathrm{dB})}=70-\operatorname{Att}_{(\mathrm{dB})} .
$$

From (7) with $L=1$, it comes that the DMT system needs a received SNR larger than $10.8 \mathrm{~dB}$ to transmit a minimal number of 2 bits per subcarrier, corresponding to a channel attenuation lower than $59.2 \mathrm{~dB}$. The following simulation results show that the SS-OFDM system can benefit from energy merging to lower the required minimal received SNR and then improve the system range.

To perform bit-loading which needs CSI at the transmitter side, we assumed that the channel is constant over one SS-OFDM symbol, that is, over $L_{t}$ OFDM symbols. This assumption cannot be valid for large values of time spreading. Furthermore, the SS-OFDM receiver have to memorize $L_{t}$ times the result of the FFT $2 \mathrm{~K}$ before any signal processing. In order to limit this memory size and to assume constant channel over one SS-OFDM symbol, the maximal time spreading is then limited to 8 .

\subsection{Single-user case}

Figures 8 and 9 give results with time spreading and $L_{f}=1$, and with frequency spreading and $L_{t}=1$, respectively. In these figures only 1D spreading is then performed. 


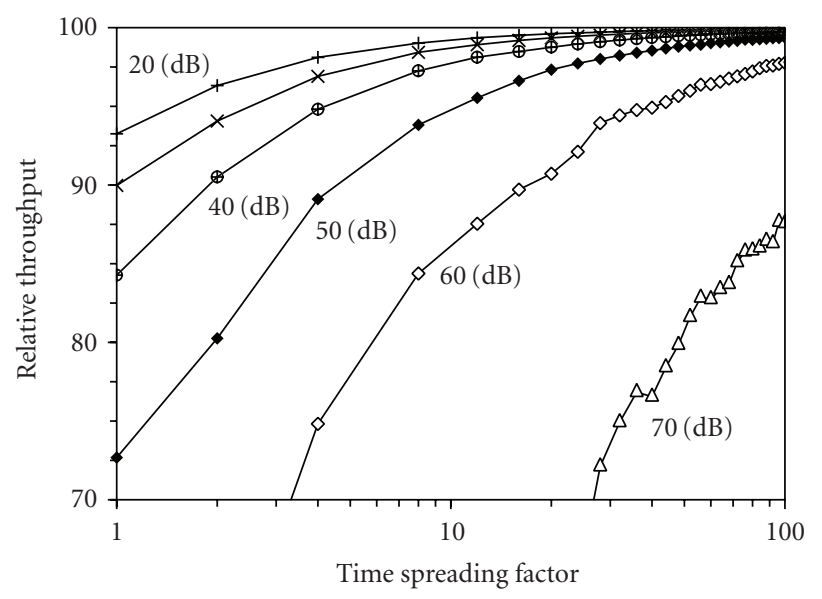

FIGURE 8: Relative throughput $R /\left(L_{t} \mathfrak{R}\right)$ in percent of SS-OFDM system with $L_{f}=1$ versus code length, over PLC channel no. 1 and for six channel attenuations $\{20,30,40,50,60,70\} \mathrm{dB}$.
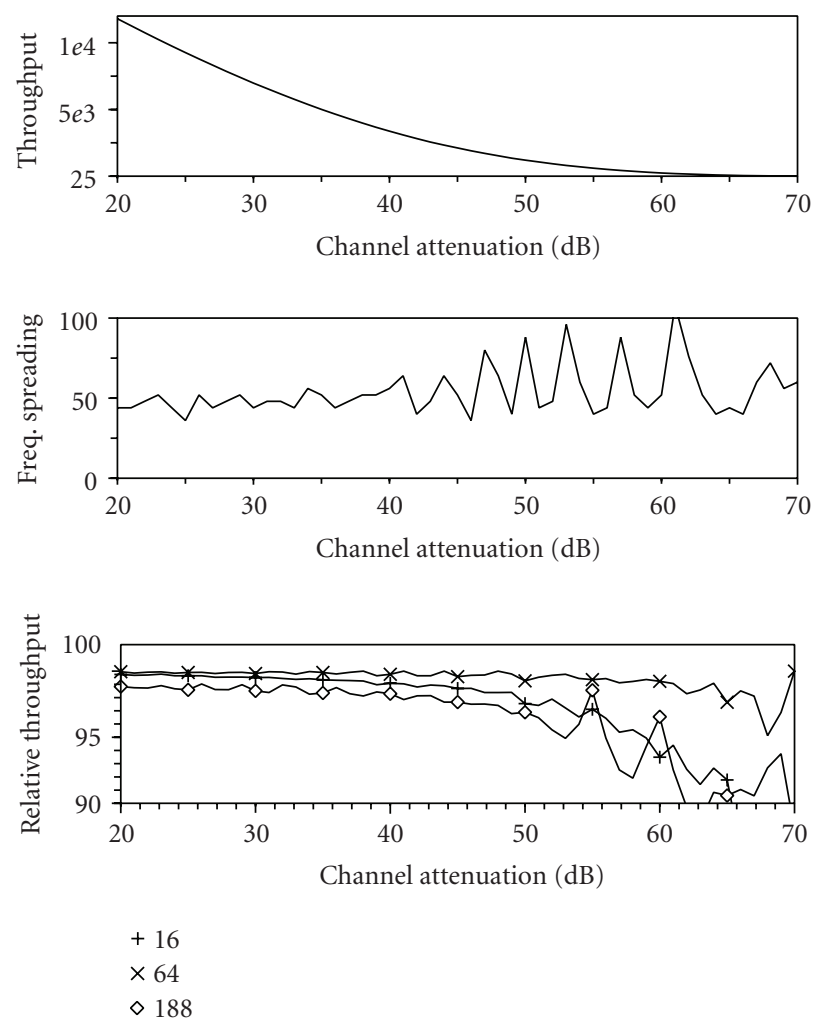

FIgURE 9: Maximal throughput $\max (R)$ in bit per SS-OFDM symbol, corresponding code length, and relative throughput $R / \max (R)$ for three code lengths $L_{f}=\{16,64,188\}$ of SS-OFDM with $L_{t}=1$ versus channel attenuation in $\mathrm{dB}$, over PLC channel no. 1 .

Figure 8 shows that the higher the channel attenuation is, the larger the time spreading factor should be to achieve a given percent of the reliable upper-bound $\mathfrak{R}$. With channel attenuation of $70 \mathrm{~dB}$, the time spreading factor must be higher than 100 to reach $90 \%$ of the reliable throughput upper-bound. For lower channel attenuations, that is, higher

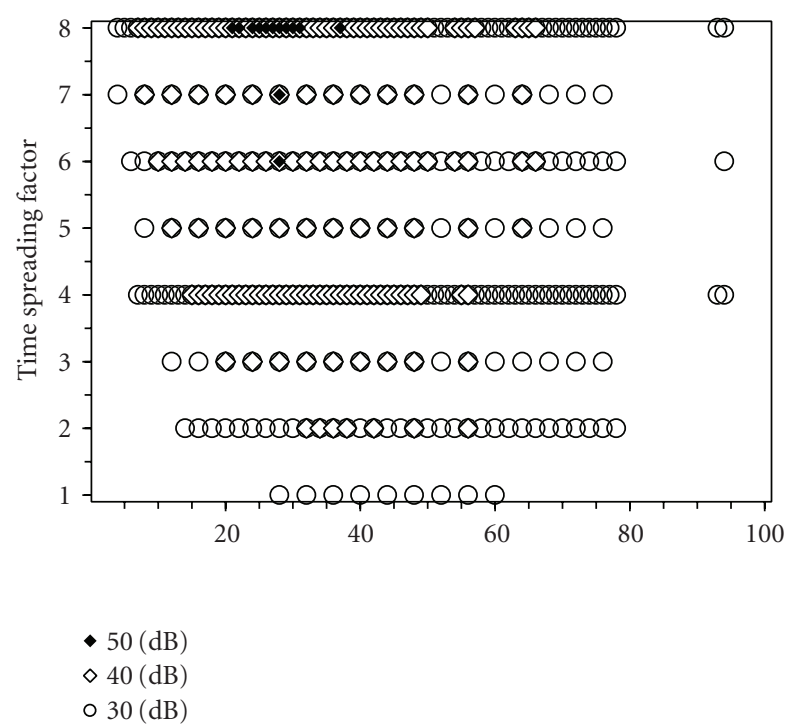

Figure 10: Time and frequency spreading configurations of SSOFDM system which lead to at least $99.5 \%$ of the maximal value of the reliable rate upper-bound $\mathfrak{R}$, over PLC channel no. 1 and for three channel attenuations $\{30,40,50\} \mathrm{dB}$.

received SNR, shorter time spreading factors are sufficient to reach a high percentage of the bound. When the channel attenuation increases, the energy per subcarrier and per OFDM symbol decreases and drops under values for which no more bits can be transmitted for an increasing number of subcarriers. To transmit bits over these zeroed subcarriers larger time spreading factors must be used to merge more OFDM symbols.

Since none analytical reliable upper-bound can be derived, as already mentioned in Section 4.2, the optimal frequency spreading factor that maximizes the throughput is worked out through simulation search. Figure 9 then shows the maximal throughput and the corresponding frequency spreading factor obtained for channel attenuations in $[20 ; 70] \mathrm{dB}$. The optimal frequency spreading is $L_{f} \in$ [36;96] with an average value around 52 . When comparing the throughput of fixed frequency spreading configurations with the optimal throughput for each channel attenuation, it appears that all the frequency spreadings, except very high $L_{f}$, give throughputs up to $99 \%$ of the maximal throughput for low channel attenuations. For high channel attenuations, the system cannot merge enough subcarriers with low $L_{f}$, and cannot compensate for the channel distortion with very high $L_{f}$ in order to improve the throughput. For these high channel attenuations, the optimal frequency spreading factor is around 64.

Figure 10 gives results with both time and frequency spreadings, that is, with $2 \mathrm{D}$ spreading. The reliable rate upper-bound $\mathfrak{R}$ is frequency spreading dependent. In order to work with a frequency spreading independent upperbound, the maximal rate upper-bound is computed over all the possible time and frequency spreading configurations, $L_{t} \leq 8$ and $L_{f} \leq 100$. Figure 10 gives the configurations 


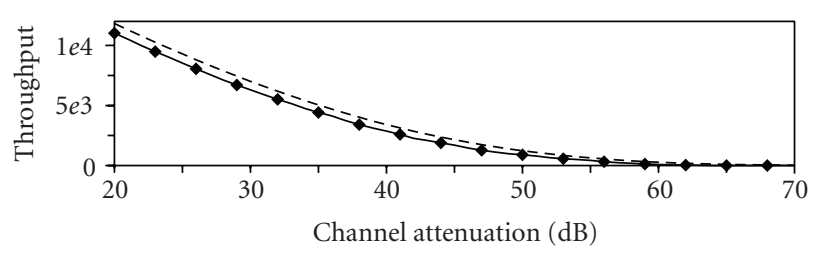

- DMT

- - - Reliable upper bound

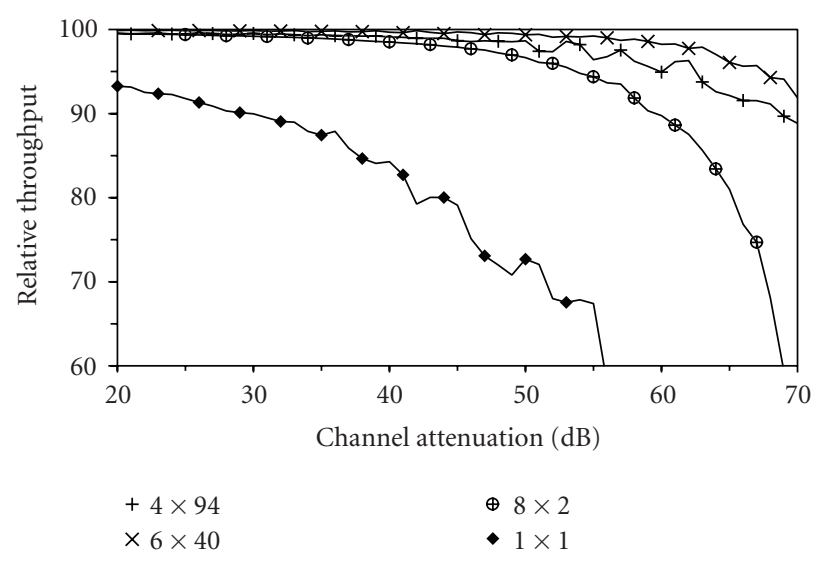

FIgURE 11: Throughput of DMT and SS-OFDM maximal reliable upper-bound in bit per OFDM symbol, relative through- put $R /\left(L_{t} \mathfrak{R}\right)$ of SS-OFDM systems in percent versus channel attenuation in $\mathrm{dB}$, with configurations $\left\{L_{t}, L_{f}\right\} \in\{\{4,94\} ;\{6,40\} ;\{8,2\}$; $\{1,1\}$.

that lead to at least $99.5 \%$ of this maximal bound. The number of these configurations decreases when the channel attenuation increases. For example, this number of configurations is equal to 285,155 , and 13 for channel attenuation, respectively, equal to 30,40 , and $50 \mathrm{~dB}$. With $50 \mathrm{~dB}$ of channel attenuation, most of the optimal configurations use a time spreading factor close to the maximal available one, that is, $L_{t}=8$. It is important to note that there exist several configurations that lead to throughputs very close to the maximal rate upper-bound. In practice, it is then possible to fix in advance, that is, not in real time but as of the system design, a subset of configurations that yield performance close to the optimal. This approach reduces the number of configurations that the system has to compare in real-time.

Figure 11 gives the throughput of the DMT system and the optimal rate upper-bound of the SS-OFDM system, and compared the throughputs of four SS-OFDM configurations relative to this upper-bound. The DMT throughput, corresponding to the SS-OFDM system with $L=L_{t}=L_{f}=1$, is easily improved by the SS-OFDM system. The DMT system cannot reach $60 \%$ of the reliable upper-bound for channel attenuations higher than $55 \mathrm{~dB}$, whereas the SS-OFDM system with $\left\{L_{t}, L_{f}\right\} \in\{\{4,94\} ;\{6,40\}\}$ can reach at least $90 \%$ of this upper-bound even for a channel attenuation equals to $70 \mathrm{~dB}$. Let us recall that for this channel attenuation the received SNR per subcarrier is lower or equal to $0 \mathrm{~dB}$. The SS-OFDM system is then able to transmit information even
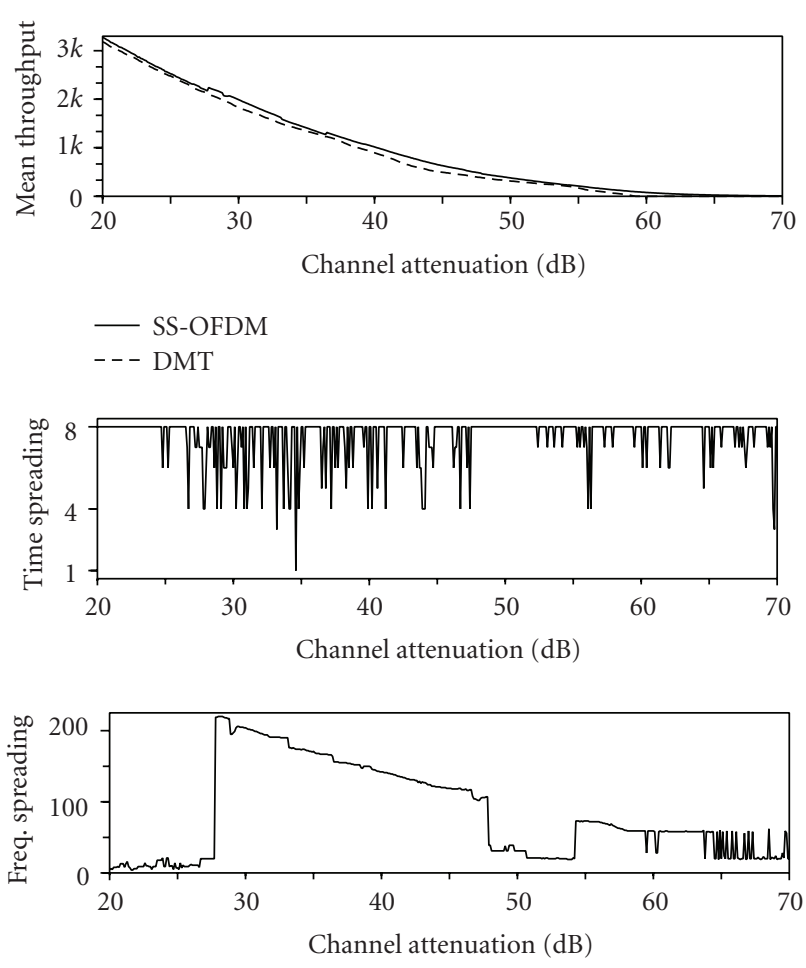

FIGURE 12: Average throughput per user of DMT and SS-OFDM, and corresponding $\left\{L_{t}, L_{f}\right\}$ SS-OFDM configuration, versus channel attenuation.

if the signal is under the noise level, whereas this is impossible with the DMT system. The SS-OFDM system can exploit subcarriers with received SNR equal to $10.8-10 \log _{10} L \mathrm{~dB}$ to transmit the lower number of bits which is 2 , that is, channel attenuation equals to $59.2+10 \log _{10} L \mathrm{~dB}$.

\subsection{Multiuser case}

In the multiuser case, the time and frequency resource is shared by the users with FDMA, whereas the SS is used to multiplex the data of each user as in the single user case. The simulation results are given for the four power line channel responses displayed in Figure 7. Each user transmits information over its own channel. Let us recall that the bitloading algorithm proposed in Section 6 aims at maximizing the smallest throughput over all users, which ensures minimal differences between their throughputs. Note that this algorithm is appropriate whatever the length code $L$, in particular for $L=1$, that is, for DMT. In order to have flexibility sharing the subcarriers $L_{f} \leq 235$ which means that the minimal number of elementary pavements per user is 2 . The maximal value of $L_{t}$ is still 8 .

Figure 12 displays the average throughput per user for the DMT and SS-OFDM systems, and the corresponding optimal configurations for the SS-OFDM system. Optimal configuration means that the time and the frequency spreading factors lead to the best maximization of the minimum users' throughput. This optimal configuration is obtained by 

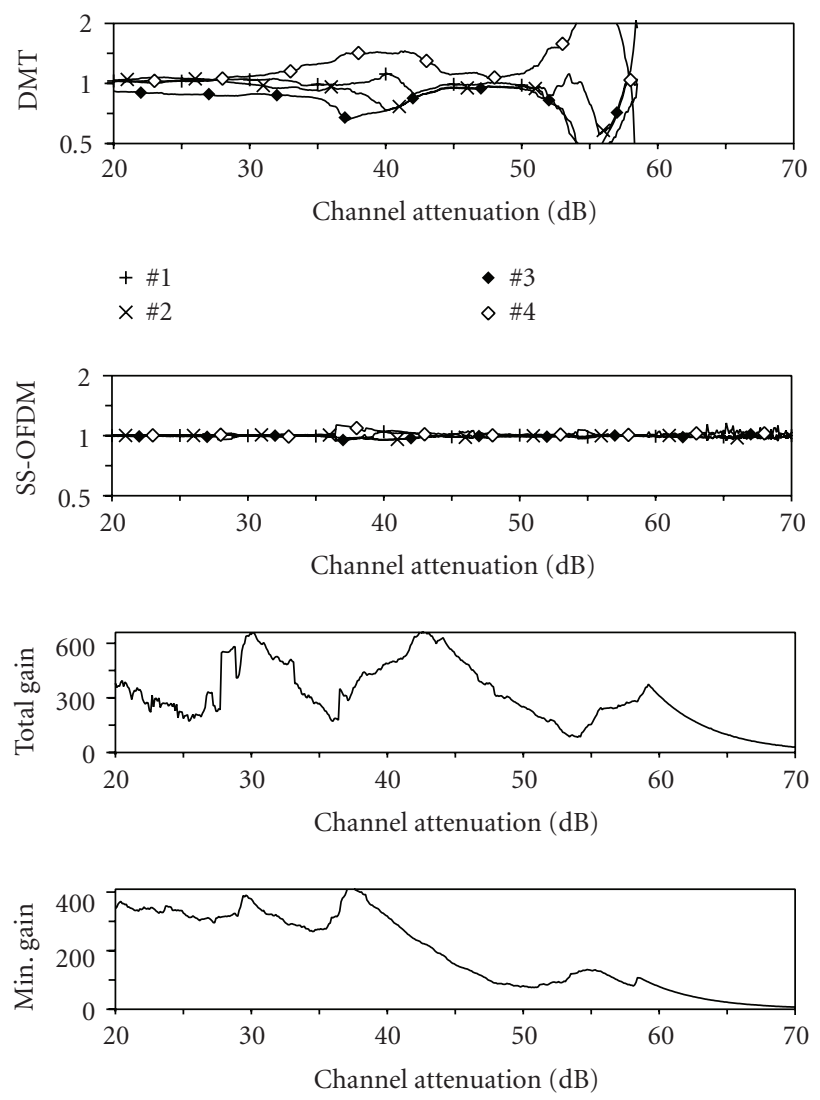

FIGURE 13: Relative throughput dispersion per user for DMT and SS-OFDM, total throughput gain, and gain of minimum user's throughput of SS-OFDM compared to DMT, versus channel attenuation.

exhaustive search over $L_{t} \leq 8$ and $L_{f} \leq 235$. The throughputs are expressed in bit per OFDM symbol, and 1 kbyte per OFDM symbol corresponds to a mean throughput per user of $9.765 \mathrm{Mbps}$ and a total throughput of $39 \mathrm{Mbps}$. For all the channel attenuations, the average throughput per user with SS-OFDM is higher than the average throughput per user with DMT, even if the multiuser bit-loading algorithm is not designed to maximize the total system throughput. The optimal time spreading factor is equal to the maximal possible value except for some particular channel attenuations and contrary to the results obtained in the single user case with $L_{f}=1$ (see Figure 8) for which the optimal time spreading factor is always the maximal possible one. As for the frequency spreading factor, Figure 12 shows that $L_{f}$ is overall decreasing with the channel attenuation from $30 \mathrm{~dB}$ but exhibits a strong dispersion of the values, $L_{f} \in[4 ; 220]$, and contrary to the single-user case with $L_{t}=1$ (see Figure 9) for which an average frequency spreading factor around 52 is obtained. Eventually, we cannot conclude that there exists a unique pair $\left\{L_{t}, L_{f}\right\}$ that yields the optimal configuration.

Figure 13 gives the throughput dispersion of each user which is evaluated as the ratio between the individual throughput and the average throughput. These values are given for the DMT system and for the SS-OFDM system

with the optimal configurations given in Figure 12. The total gain is the difference between the SS-OFDM and DMT total throughputs. The minimum gain is the difference between the SS-OFDM and DMT minimal throughputs among users. As evident from the obtained results, the SS-OFDM system provides a lower throughput dispersion between users than DMT. The adaptive SS-OFDM system has in fact much more variable parameters than DMT, which allows to better answer to the resource sharing problem, and especially minimize the throughput dispersion. Now focusing on the throughput gain, the total and the minimal user throughputs are improved with SS-OFDM. In the figure, a gain of 300 bits per OFDM symbol corresponds to a gain of $2.9 \mathrm{Mbps}$. Then, for channel attenuation equals to $50 \mathrm{~dB}$, the minimum throughput obtained with $\left\{L_{t}, L_{f}\right\}=\{8,31\}$ is increased by $779 \mathrm{bps}$, that is, $27 \%$, and the corresponding total gain is $20 \%$. For lower channel attenuations, for example $30 \mathrm{~dB}$, the minimum throughput is increased by $23 \%$, and the total throughput by $9 \%$. For all the channel attenuations the gain of the minimum throughput is higher than $11.8 \%$.

Complementary simulations show that there exist 175 configurations that give a gain of at least $10 \%$ for all the channel attenuations, and 449 configurations for which SSOFDM outperforms DMT for all the channel attenuations. Then, there is a great choice of configurations that allow to achieve higher rates than DMT. To simplify this choice, Table 1 proposes optimal spreading configurations for some ranges of channel attenuations. Note that all users can transmit low rate information up to $83 \mathrm{~dB}$ of channel attenuation with SS-OFDM, whereas DMT needs channel attenuations lower than $58 \mathrm{~dB}$ to transmit information for all users. For example, for the largest range, that is, channel attenuation in $[20 ; 83]$, the optimal configuration is $\left\{L_{t}, L_{f}\right\}=\{8,5\}$ yielding a minimum throughput gain of $11.8 \%$ (the minimal value is obtained for $20 \mathrm{~dB}$ of channel attenuation). A minimum gain of $26.4 \%$ is reached for channel attenuations higher than $50 \mathrm{~dB}$. These results show that it is possible in practice to select a small number of interesting configurations depending on channel attenuation ranges to reach throughputs higher than those obtained with DMT. More generally, it is possible to pick and choose a set of interesting configurations according to all the practical transmission scenarios that will be used to design the modem. The online modem will only have to select in real time the configuration adapted to its transmission link.

\section{CONCLUSION}

In this paper, we have studied adaptive loading principles applied to SS-OFDM systems suitable for wireline networks in a multiple-user context. A practical loading algorithm based on a $2 \mathrm{D}$ energy merging strategy has been proposed to maximize the system throughput and the system range. The adaptive SS-OFDM system offers allocation flexibility due to a large number of variable parameters. These parameters have been optimized through analytical studies and simulations, at a target symbol error rate and under PSD constraint. The 2D spreading component of the SS-OFDM system translates 
TABLE 1: SS-OFDM optimal configuration and minimum user's throughput gain versus channel attenuation.

\begin{tabular}{c|cc}
\hline $\begin{array}{c}\text { Chan. at. } \\
(\mathrm{dB})\end{array}$ & $\begin{array}{c}\text { Opt. config. } \\
\left(L_{t} \times L_{f}\right)\end{array}$ & $\begin{array}{c}\text { Min. user's gain } \\
(\%)\end{array}$ \\
\hline $20-83$ & $8 \times 5$ & 11.8 \\
$30-83$ & $8 \times 15$ & 15.0 \\
$20-30$ & $8 \times 5$ & 11.8 \\
$30-40$ & $8 \times 191$ & 18.7 \\
$40-50$ & $8 \times 38$ & 23.3 \\
$50-58$ & $8 \times 31$ & 26.4 \\
\hline
\end{tabular}

into energy merging in the time and frequency domains that leads to an increase of the throughputs compared with the classical DMT approach. This gain is understood as a reduction of the quantification loss due to the finite-order modulations. Well-chosen spreading factors lead to minimum user throughputs improved by at least $11.8 \%$ for low channel attenuations, and more than $23.3 \%$ for channel attenuations higher than $40 \mathrm{~dB}$. Among the large number of possible spreading configurations, it is possible to establish a limited number of system configurations adapted to pratical transmission scenarios, and chosen in real time by the online modem.

\section{ACKNOWLEDGMENTS}

The authors would like to thank the anonymous reviewers for their insightful comments. This paper has been presented in part at the IEEE Global Communications Conference, San Francisco, 2006, and at the IEEE International Symposium on Spread Spectrum Techniques and Applications, ManausAmazomn, 2006.

\section{REFERENCES}

[1] L. H.-J. Lampe and J. B. Huber, "Bandwidth efficient power line communications based on OFDM," International Journal of Electronics and Communications, vol. 54, no. 1, pp. 2-12, 2000.

[2] E. Del Re, R. Fantacci, S. Morosi, and R. Seravalle, "Comparison of CDMA and OFDM techniques for downstream powerline communications on low voltage grid," IEEE Transactions on Power Delivery, vol. 18, no. 4, pp. 1104-1109, 2003.

[3] J. Cioff, "A multicarrier primer," Tech. Rep. T1E1.4/91-157, ANSI, Washington, DC, USA, committee contribution, 1991.

[4] G. D. Forney Jr. and L.-F. Wei, "Multidimensional constellations - part I: Introduction, figures of merit, and generalized cross constellations," IEEE Journal on Selected Areas in Communications, vol. 7, no. 6, pp. 877-892, 1989.

[5] O. Isson, J.-M. Brossier, and D. Mestdagh, "Multi-carrier bit-rate improvement by carrier merging," Electronics Letters, vol. 38, no. 19, pp. 1134-1135, 2002.

[6] S. Mallier, F. Nouvel, J.-Y. Baudais, D. Gardan, and A. Zeddam, "Multi-carrier CDMA over copper lines-Comparison of performances with the ADSL system," in Proceedings of the 1st International Workshop on Electronic Design, Test \& Applications (DELTA '02), pp. 450-452, Christchurch, New Zealand, January 2002.
[7] M. Crussière, J.-Y. Baudais, and J.-F. Hélard, "adaptive linear precoded DMT as an efficient resource allocation scheme for power-line communications," in Proceedings of IEEE Global Telecommunications Conference (GLOBECOM '06), pp. 1-5, San Francisco, Calif, USA, November 2006.

[8] M. Crussière, J.-Y. Baudais, and J.-F. Hélard, "Improved throughput over wirelines with adaptive MC-DS-CDMA," in Proceedings of the 9th IEEE International Symposium on Spread Spectrum Techniques and Applications, pp. 143-147, Manaus, Amazon, Brazil, August 2006.

[9] M. Crussière, J.-Y. Baudais, and J.-F. Hélard, "Adaptive spreadspectrum multicarrier multiple-access over wirelines," IEEE Journal on Selected Areas in Communications, vol. 24, no. 7, pp. 1377-1388, 2006.

[10] G. B. Giannakis, P. A. Anghel, and Z. Wang, "Generalized multicarrier CDMA: Unification and linear equalization," EURASIP Journal on Applied Signal Processing, vol. 2005, no. 5, pp. 743-756, 2005.

[11] A. S. Hedayat, N. J. A. Sloane, and J. Stufken, Orthogonal Arrays: Theory and Applications, chapter 7, Springer, New York, NY, USA, 1999.

[12] S. Hara and R. Prasad, "Overview of multicarrier CDMA," IEEE Communications Magazine, vol. 35, no. 12, pp. 126-133, 1997.

[13] S. Kondo and L. Milstein, "On the use of multicarrier direct sequence spread spectrum systems," in Proceedings of IEEE Military Communications Conference (MILCOM '93), vol. 1, pp. 52-56, Boston, Mass, USA, October 1993.

[14] N. Yee, J.-P. Linnartz, and G. Fettweis, "Multi-carrier CDMA in indoor wireless radio networks," in Proceedings of the International Symposium on Personal, Indoor and Mobile Radio Communications (PIMRC'93), pp. 109-113, Yokohama, Japan, September 1993.

[15] S. Kaiser and K. Fazel, "A flexible spread-spectrum multicarrier multiple-access system for multi-media applications," in Proceedings of the 8th IEEE International Symposium on Personal, Indoor and Mobile Radio Communications (PIMRC '97), vol. 1, pp. 100-104, Helsinki, Finland, September 1997.

[16] C. Y. Wong, R. S. Cheng, K. B. Letaief, and R. D. Murch, "Multiuser OFDM with adaptive subcarrier, bit, and power allocation," IEEE Journal on Selected Areas in Communications, vol. 17, no. 10, pp. 1747-1758, 1999.

[17] T. Sartenaer, L. Vandendorpe, and J. Louveaux, "Balanced capacity of wireline multiuser channels," IEEE Transactions on Communications, vol. 53, no. 12, pp. 2029-2042, 2005.

[18] W. Rhee and J. M. Cioffi, "Increase in capacity of multiuser OFDM system using dynamic subchannel allocation," in Proceedings of the 51st IEEE Vehicular Technology Conference (VTC '00), vol. 2, pp. 1085-1089, Tokyo, Japan, May 2000.

Jean-Yves Baudais received the M.S. degree, and $\mathrm{Ph} . \mathrm{D}$. degree in electrical engineering from the National Institute of Applied Sciences of Rennes (INSA), France, in 1997 and 2001, respectively. Since 2002, he has been working as a CNRS researcher (French National Centre for Scientific Research) in the Institute for Electronics and Telecommunications of Rennes (IETR). His current research focuses on transmitter and receiver diversity techniques for multiuser and multicarrier communication including space-time coding. 
Matthieu Crussière received the M.S. and Ph.D. degrees in electrical engineering from the National Institute of Applied Sciences (INSA), France, in 2002 and 2005, respectively. During his Ph.D. degree, he was with the Electronics and Telecommunications Institute of Rennes (IETR), where he worked on the optimization of high bit rate power line communications. Since 2005, he has been an Assistant Professor in the De-

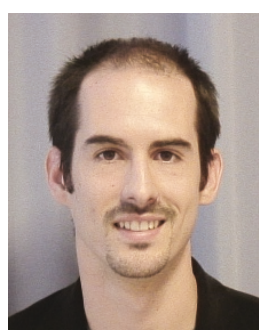
partment of Telecommunications and Electronic Engineering at INSA and currently pursues his research activities at IETR. His main research interests lie in digital communications and signal processing techniques, and particularly focus on multicarrier spread-spectrum systems, synchronization, channel estimation, and adaptive resource allocation. He has been involved in several European and national research projects including power line communications, ultra-wideband systems, and mobile radio communications. 